\title{
A New Type of Hybrid Semi-Active Control Strategy in the Application of the High-speed Railway Vehicle Vibration Control
}

\author{
Tiansheng $\mathrm{Gu}^{1, \mathrm{a}}$ \\ ${ }^{1}$ Xi an Railway Vocational\&Technical Institute, 710014 \\ ${ }^{\mathrm{a}}$ email
}

Keywords: The Semi-Active Control Strategy; SH-ADD; Application

\begin{abstract}
. the article firstly introduces two main high-speed locomotive vehicle semi-active control strategy, and aims at SH-ADD a new hybrid semi-active control strategy are discussed in this paper From the point of the research results of the example analysis, the SH-ADD hybrid semi-active control strategy has obvious superiority, realize the effective control of low frequency and intermediate frequency range, can effectively reduce the lateral stability index, at the same time also can reduce the body lateral acceleration.
\end{abstract}

\section{Introduction}

Usually greater than the speed of $200 \mathrm{~km} / \mathrm{h}$ speed of passenger and EMU consisting of high-speed railway vehicles, due to the high speed railway vehicle running speed is faster, so the vehicle is in operation vibration is very large, which is particularly severe lateral vibration. To reduce this vibration, to ensure high-speed iron safe and comfortable operation of the vehicle, usually semi-active suspension control system to control.

\section{A Semi-active Suspension System}

High-speed railway vehicle running smooth and comfort need to train suspension control system controlled by the suspension system optimization design, to achieve effective attenuation of lateral vibration. High-speed railway applications listed three main suspension system comprises passive suspension system, all active suspension system with semi-active suspension system. Because the passive suspension system spring element and the damping element parameters are not adjustable, so there is a big limitation to ensure the smooth operation of the train, etc., and full active suspension system because of its high cost and complexity, in fact, difficult to be applied. Compared with full active suspension system, semi-active suspension system lower prices, simple, and therefore has broad prospects for development.

Semi-active suspension system is comprised of a controller, sensor devices and semi-active damper composition, the role of the controller is to get feedback signal from the sensor is calculated to obtain the required damping force, wherein the feedback signal from the sensing means Being semi-control strategy. After calculating the damping force shock absorber controller will issue execution on shock absorbers, semi-active damper drive magnetic or electromagnetic valve according to the instructions made by the appropriate adjustments. The control technology from the research point of view, China is still confined to the theoretical analysis and laboratory tests, and has been successfully used in foreign commerce. Whether semi-active suspension system can be applied in practice, depending on the control strategy and control dampers, and current research on against both carried out.

\section{The Semi-active Control Strategy}

On semi-active control strategy point of view the main stage RS control, SH skyhook damping control and ADD acceleration control simple semi-active control strategy, as well as adaptive control, fuzzy control and neural network control and other complex modern half active control strategy, which the simple semi-active control strategy more commonly used in commercial 
applications with trials, it not only can be manually adjusted, but also with a sensor control device automatically adjusts the instrument constituting the system. Now high-speed railway vehicle $21 / 4$ car of its own suspension system lateral model skyhook damping control and acceleration control strategy analysis.

Skyhook semi-active control strategy proposed by Karnopp, the control strategy and the control system is not much relationship, robust, and simple control strategy. The switch control strategy into specific semi-active and continuous semi-active, and is widely used in practical engineering.

SH skyhook semi-active control strategy expression:

$$
c=\left\{\begin{array}{l}
c_{\max } \dot{y}\left(\dot{y}-\dot{y}_{t}\right) \geq 0 \\
c_{\min } \dot{y}\left(\dot{y}-\dot{y}_{t}\right)<0
\end{array}\right.
$$

Wherein $\dot{y}, \dot{y}_{t}$ the sprung mass and unsprung mass speed; $c_{\max }, c_{\min }$ are the maximum and minimum damping.

Skyhook semi-active control by the vehicle speed is proportional to the absolute control, to achieve the acceleration of the vehicle body vibration reduction, since it is difficult to measure the absolute speed of the vehicle body, and therefore is generally used to measure vehicle acceleration by the acceleration sensor and then get the absolute velocity of the vehicle body. But this one inevitable errors exist, such as the sensor itself and system noise and other errors will cause error absolute velocity of the vehicle body, the presence of these errors will affect the damping effect. In the practical application of this body can not be installed over the damper, but the increase in the role of a skyhook simulation system, capable of generating the active force element to achieve a similar damping effect.

ADD acceleration damping plate is the use of active control strategy that is proportional to the acceleration of the vehicle body vibration damping force to suppress the vibration of the vehicle body.

ADD acceleration semi-active damping control strategy expression:

$$
c=\left\{\begin{array}{l}
c_{\max } \ddot{y}\left(\dot{y}-\dot{y}_{t}\right) \geq 0 \\
c_{\min } \ddot{y}\left(\dot{y}-\dot{y}_{t}\right)<0
\end{array}\right.
$$

Wherein $\dot{y}, \dot{y}_{t}$ the sprung mass and unsprung mass speed; $c_{\max }, c_{\min }$ are the maximum and minimum damping. ADD acceleration semi-active damping control strategy of the acceleration sensor is not sensitive to the error, with semi-active control over a wider frequency domain skyhook control, the control is better, the vehicle vibration can be effectively damped, thus improving railway locomotive smoothness and comfort.

1/4 sports car model differential equation can be expressed:

$$
\left.\begin{array}{l}
m_{s} \ddot{y}+k\left(y-y_{t}\right)+c\left(\dot{y}-\dot{y}_{t}\right)=0 \\
m_{t} \ddot{z}_{t}+k\left(y_{t}-y\right)+k_{t}\left(y_{t}-y_{r}\right)+c\left(\dot{y}_{t}-\dot{y}\right)=0
\end{array}\right\} \text { or }
$$

Where $m_{s}, m_{t}$ are the mass of the vehicle body and the bogie, unit kg; respectively, $k, k_{t}$ a beam line and two-line rigidity units $\mathrm{N} \bullet \mathrm{m}^{-1}$; c is the secondary lateral damping unit $\mathrm{N} \bullet \mathrm{s} \bullet \mathrm{m}-1$.

The equation is transformed into a 1/4 suspension Simulink model, as shown in Figure 1. 


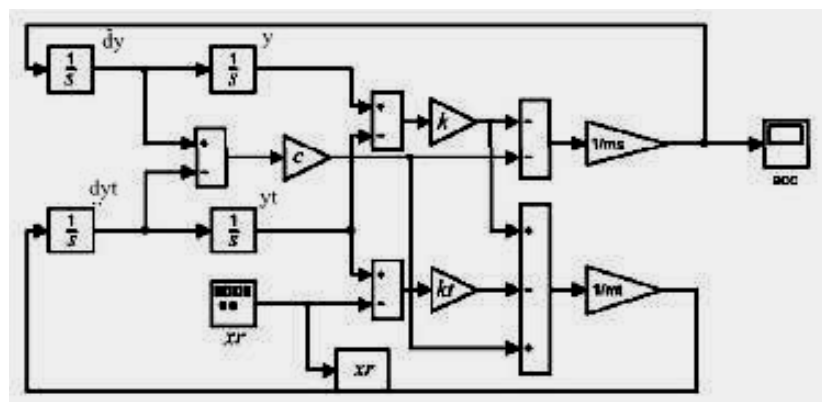

To SH and ADD two semi-active control strategy compared acceleration transmissibility, Savaresi, who used a non-linear transfer rates approximate expression:

$$
\hat{F}_{a c c}\left(j \omega_{i}\right)=\sqrt{\frac{\frac{1}{T} \int_{0}^{T}\left(\ddot{y}_{i}(t)^{2}\right) d t}{\frac{1}{T} \int_{0}^{T}\left(y_{r i}(t)^{2}\right) d t}}(i=1,2, \ldots, N)
$$

Which are single-frequency excitation amplitude and acceleration of the vehicle at this time.

By using sinusoidal excitation of $\mathrm{SH}$, ADD control strategies vehicle acceleration transmissibility is described to obtain Fig.2

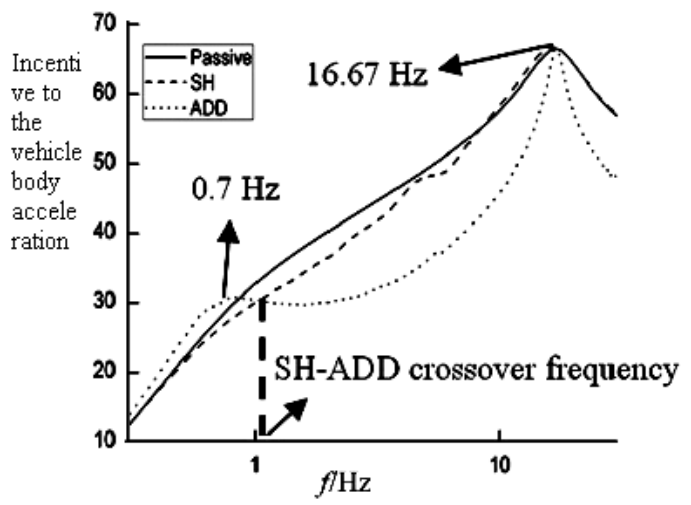

Figure $2 \mathrm{SH}$, transfer rate of the vehicle body acceleration ADD under control strategy

Wherein $y_{t}(t)=a \sin (2 \pi f t), a=0.05 m, 0.2 H Z<f<20 H Z$ the length of the simulation time is set to $100 \mathrm{~s}$, in steps of $0.001 \mathrm{~s}$. According to Figure 3 was found in the frequency range below the second order of $16.67 \mathrm{HZ}$, SH skyhook semi-active control strategy is effective, but above the second order frequency range, $\mathrm{SH}$ and quite passive control strategy; in below the first order frequency $0.7 \mathrm{HZ}$, ADD acceleration semi-active damping control strategy is not ideal, but still higher than the passive control, but higher than the first-order frequency control effect ADD significantly better. On the other hand, from the point of view Figure $3 \mathrm{SH}$ control effect and ADD control effect the formation of a complementary form, and $1.1 \mathrm{HZ}$ as a dividing point, when below $1.1 \mathrm{HZ}$, SH control is excellent, and when the above 1.1HZ ADD when control effect of better. On this basis, Savaresi et al proposed a new semi-active control strategy, namely SH-ADD hybrid control strategy.

\section{SH-ADD Mixing Semi-active Control Strategy}

Savaresi of SH et al skyhook semi-active control strategies and mixing ADD acceleration damping control of semi-active control strategy SH-ADD, the control strategy only need to use one acceleration sensor can be realized. 


$$
\left\{\begin{array}{l}
C_{\max }\left[\left(\ddot{y}^{2}-\alpha^{2} \dot{y}^{2}\right) \leq 0 \& \& \dot{y}\left(\dot{y}-\dot{y}_{t}\right)>0\right] \| \\
{\left[\left(\ddot{y}^{2}-\alpha^{2} \dot{y}^{2}\right)>0 \& \& \dot{y}\left(\dot{y}-\dot{y}_{t}\right)>0\right]} \\
C \min \left[\left(\ddot{y}^{2}-\alpha^{2} \dot{y}^{2}\right) \leq 0 \& \& \dot{y}\left(\dot{y}-\dot{y}_{t}\right) \leq 0\right] \| \\
{\left[\left(\ddot{y}^{2}-\alpha^{2} \dot{y}^{2}\right)>0 \& \& \dot{y}\left(\dot{y}-\dot{y}_{t}\right) \leq 0\right]}
\end{array}\right.
$$

Wherein, \&\& is the logical relationship "and" logical relationship "or" transform coefficients, the transition conditions. When the transition condition is greater than 0 , the size of the ADD damping control strategy control, when the transition condition $\leqslant 0$, the damping control strategy to control by SH, SH-ADD hybrid control strategy to control the effect of the decision by the conversion factor, The conversion factor by the displacement signal decision.

Suppose displacement signal and velocity terms are as follows:

$$
\begin{gathered}
x(t)=a \sin (a \pi f t), \dot{x}(t)=z \pi f \cos (2 \pi f t) \\
a=2 \pi f=2 \pi f_{\text {cross }}
\end{gathered}
$$

Wherein $f_{\text {cross }}$ is the SH control and frequency control ADD intersection.

Figure 3 shows the cross point of the case is $1.1 \mathrm{HZ}$, calculated according to the above formula was converted coefficient $2.2 \pi$, the conversion factor into the SH-ADD-generation hybrid control strategy expression, you can get 2 1/4 degrees of freedom model of car hybrid control strategy. On the transfer characteristic SH-ADD hybrid control strategy analysis found that the mitigation strategies set the SH and ADD control of both the advantages and the disadvantages of each removal, the hybrid control strategy transfer characteristics at low frequencies similar to SH control, high-frequency with ADD when control passed similar characteristics, it combines the advantages of both.

\section{Modeling and Simulation}

To explore the SH-ADD mixing semi-active control applications strategy, magnetorheological damper EMU Yongqiang here to establish a semi-active control of the EMU train simulation model and an example.

For simulation, Liu Yongqiang, etc. were established using ADAMS high speed railway vehicle model, combined with the non-linear suspension (a line, two lines), random orbital excitation, nonlinear factors and other contact with the track starting to build Motor Car trailer special model, and lateral secondary suspension damper using SH-ADD hybrid control strategy, and then build a new hybrid semi-active control system, bogie shown in Figure 3. EMU steering model comprising framework, a series of suspension system (Fig. 4), the secondary suspension system (Fig. 5), wheel, traction links, traction motors and drive systems and other structures. Wherein the secondary suspension system for the lateral limits of its lateral displacement stopper capable of traveling is in the vehicle curve relative displacement transverse stopper once more than the allowable range, the stopper will play a role in prompting the body to restore balance.

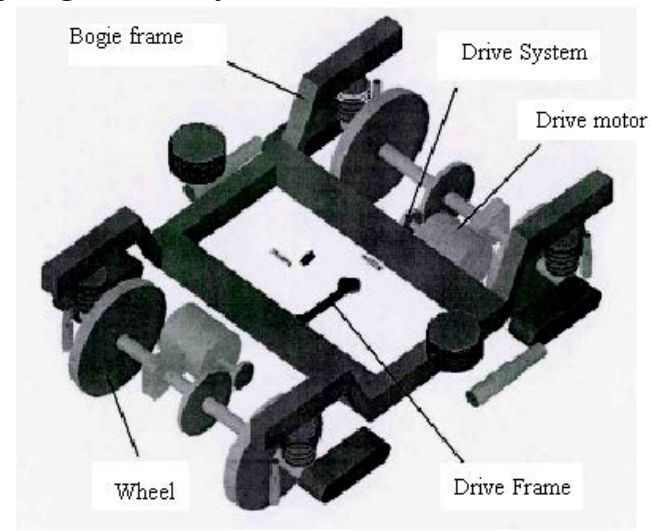

Figure 3 Bogie 


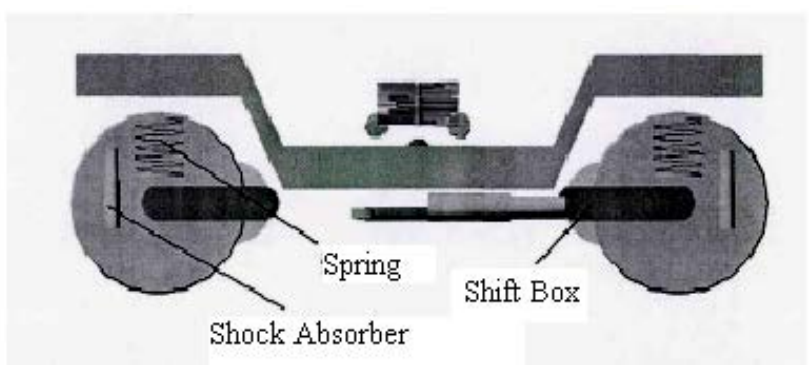

Figure 4 a Bogie Suspension

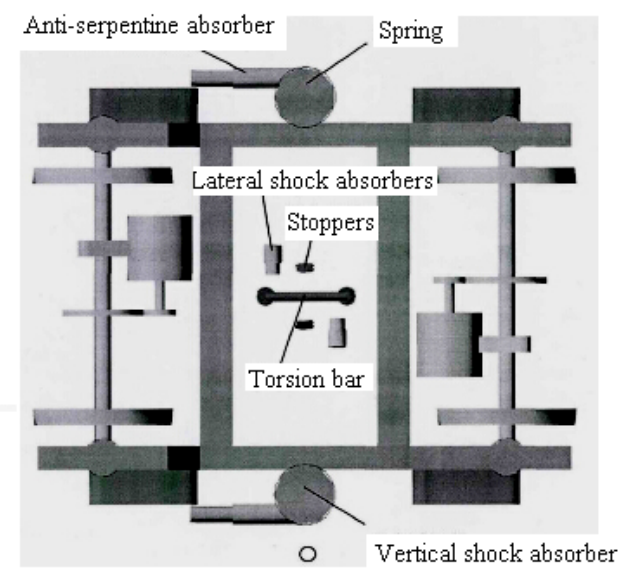

Figure 5 Bogie secondary suspension

Liu Yongqiang built up on the basis of the use of ADAMS vehicle model, the establishment of a bicycle model, through the secondary suspension of the bogie, traction rod combined with the body forms a bicycle model, then the model from cycling through the buffer coupler connection (Fig. 6), the establishment of a high-speed EMU train model. Train model is built with a total of eight cars, section 6 of the trailer. On the power of the traditional assembly of centralized and decentralized two. In addition, also we have established a wheel/rail contact model and track Incentive Model.

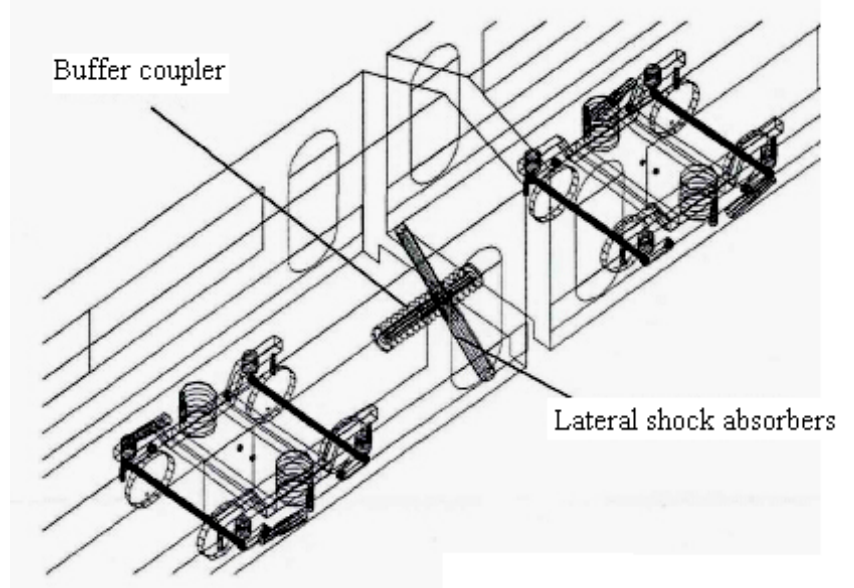

Figure 6 buffer coupler lateral damper and workshop

Simulation Analysis of mainly sports performance stability, smooth operation, security, and curve for evaluation, a German low interference orbit Cape simulation analysis of semi-active control, the results show a mixed semi-active control system is capable of great reduce the degree of lateral vibration of the vehicle body, to ensure the smooth operation of the vehicle. Compared with passive control, SH control, ADD control, control performance SH-ADD hybrid semi-active control of the best, not only can effectively reduce the lateral stability index, while reducing the lateral acceleration of the vehicle body.

\section{Conclusion}

SH-ADD text from the semi-active control strategy, introduces new SH-ADD mixing semi-active 
control strategy established by analyzing the characteristics found mixed with semi-active control strategy compared to traditional control has better control effect, by SH, ADD two kinds of semi-active control strategy combining the advantages of both to achieve the complementary advantages, disadvantages abandoned, resulting in low frequency, high frequency can be effectively controlled. By analyzing the examples, the new SH-ADD mixing semi-active control strategy simulation found that the system is to a large extent reduce the lateral acceleration of the vehicle body and lateral vibration of the vehicle body, can effectively guarantee the smooth running of the vehicle body.

\section{References}

[1] Liao Yingying, Liu Yongqiang, Yang Shaopu. A new hybrid semi-active control strategy in high speed railway vehicle vibration control [J]. Vibration and shock, 2013,12: 84-87.

[2] Liao Yingying. High-speed railway vehicle semi-active suspension system dynamics modeling optimization and simulation analysis [D]. Beijing Jiaotong University, 2012.

[3] Ma Xinna. transverse vibration control and kinetics of [D]. Based on MR damper high-speed locomotives. Beijing Jiaotong University, 2012.

[4] Ma Lu based on negative capacitance of the piezoelectric shunt damping circuit main-Passive Hybrid vibration of [D]. control Nanjing University of Aeronautics and Astronautics, 2012.

[5] Li Jizhong. based on half-speed train stability and vibration characteristics of the active suspension system research [D]. Southwest Jiaotong University, 2013.

[6] Liu Yongqiang. Based on magnetorheological damper EMU semi-active control and Delay Analysis [D]. Beijing Jiaotong University, 2011.

[7] Zong Luhang. MR damper dynamics model and its application in vehicle suspension of [D]. University of Science and Technology of China, 2013.

[8] Wang Xiaozhong. Speed train lateral semi-active damper design and research [D]. Southwest Jiaotong University, 2011. 